\title{
Correction: Reactive Searching and Infotaxis in Odor Source Localization
}

The PLOS Computational Biology Staff

\section{Notice of Republication}

This article was republished on October 28, 2014, to correct an error in displaying the PDF. Please download the PDF again to view it correctly. The previous version of the PDF is not available because an error prevented it from being viewed.

\section{Reference}

1. Voges N, Chaffiol A, Lucas P, Martinez D (2014) Reactive Searching and Infotaxis in Odor Source Localization. PLoS Comput Biol 10(10): e1003861. doi:10.1371/journal.pcbi.1003861
Citation: The PLOS Computational Biology Staff (2014) Correction: Reactive Searching and Infotaxis in Odor Source Localization. PLoS Comput Biol 10(11): e1004019. doi:10.1371/journal.pcbi.1004019

Published November 17, 2014

Copyright: (๑) 2014 The PLOS Computational Biology Staff. This is an openaccess article distributed under the terms of the Creative Commons Attribution License, which permits unrestricted use, distribution, and reproduction in any medium, provided the original author and source are credited. 